\title{
Incidencia del suicidio consumado en el partido judicial de San Vicente del Raspeig (Alicante).
}

\author{
Consummated suicide rate in the judicial district of \\ San Vicente del Raspeig (Alicante).
}

\section{G. Ayala Espinosa ${ }^{1}$, JB. Martí Lloret ${ }^{1}$ y F. Rodes Lloret ${ }^{1}$}

\section{RESUMEN}

OBJETIVOS: I.- Determinar el número de casos de suicidio consumado en San Vicente del Raspeig entre 1990 y 200I. 2. Describir las características, circunstancias y factores de riesgo asociados.

METODOLOGÍA: Se trata de una investigación con diseño de serie temporal compuesta de un conjunto de diez estudios transversales anuales y consecutivos en el periodo 1990-200I. Como fuente de obtención de datos se estudian las diligencias previas de los archivos de los juzgados del Partido Judicial San Vicente del Raspeig. Las variables a considerar son: género, edad, estado civil, mecanismo, lugar del suicidio, municipio dónde tuvo lugar, año del suicidio, intentos anteriores y documentos dejados. Se ha utilizado el test chicuadrado y un nivel de significación preestablecido $(p<0,05)$.

RESULTADOS: El número de suicidios es de 92 . Se suicidan más hombres (63) que mujeres (29) con una proporción de 2,17:I. En todas las edades encontramos hombres que se suicidan. No encontramos mujeres que se suiciden menores de $\mathbf{3 0}$ años. Los hombres entre 50-59 y 70-79 y las mujeres de la década de los 60 son los que más consuman los hechos $(\mathrm{p}=0,040)$. El estado civil más frecuente es el de casado con 39 casos, 28 hombres $(56 \%$ y II mujeres $(45,83 \%)$. Los hombres eligen preferentemente la ahorcadura $(44,44 \%)$, las mujeres la precipitación $(51,72 \%)(p=0,043)$. Los hombres prefieren terminar con su vida en el propio domicilio $(46,03 \%)$, las mujeres en la vía pública $(34,48 \%)(p=0,006)$. Los hombres eligen para quitarse la vida preferentemente el municipio de Campello $(31,75 \%$ ) y San Vicente $(25,40 \%)$. Las mujeres Campello $(27,59 \%$ y San Juan $(24,14 \%)$. En el $92,31 \%$ de las mujeres se encuentra antecedentes de intentos de suicidio, en los hombres el $50 \%(p=0,010)$. El $26,98 \%$ de los hombres dejan documentos, en las mujeres encontramos en el $6,90 \%(p=0,027)$.

CONCLUSIONES: El género masculino y la edad de 50 a 70 años se asocia a riesgo más elevado de suicidio. Los hombres prefieren mecanismos más elaborados para suicidarse, en su domicilio y dejar constancia en algún documento. En las mujeres hay más intentos de suicidio, no encontramos suicidas menores de $\mathbf{3 0}$ años, y cuando se deciden se quieren asegurar de no fallar mediante la precipitación en vía pública. Consideramos el incremento de la edad como factor asociado al suicidio.

Palabras clave: Suicidio consumado, incidencia, factores de riesgo.

Cuad Med Forense 2005; 11(40):119-129
ABSTRACT

OBJECTIVES: L- - To determine the number of consummated suicides in San Vicente del Raspeig between 1990 and 2001 . 2.- To describe the characteristics, circumstances and associated risk factors.

METHODOLOGY: It is an investigation of a temporary series made of a sample of ten annual studies between 1990-2001. We used as a source the previous investigations found in the archives of the courts of San Vicente del Raspeig (Alicante). The variables to consider are: gender, age, marital status, mechanism, place of suicide, county where it took place, year of suicide, number of attempts previousl and notes left. We used the test as "chi-cuadrado" and a previous determined significance level $(p<0.05)$.

RESULTS: The number of suicides is 92 . More men (63) than women (29) commit suicide with a proportion of 2. 17: I. In all ages we found men that committed suicide. We did not find any women that committed suicide under the age of 30 . Men between 50-59 and 70-79 and women around 60 are in the highest rate $(p=0.040)$. Married people commit suicide more than single people with 39 cases, of which 28 are men (56\%) and I I women (45.83\%). Men prefer hanging (44.44\%) and women jumping $(51.72 \%)(p=0.043)$. Men prefer to commit suicide at home (46.03\%) and women on a public place $(34.48 \%)(p=0.006)$. Men Prefer to do it in El Campello (31.75\%) and San Vicente (25.40\%) and women in El Campello (27.59\%) and San Juan $(24.14 \%)$. Women try more times $(92.31 \%)$ than men $(50 \%)$ $(p=0.010)$. Men leave more suicide notes $(26.98 \%)$ than women $(6.90 \%)(p=0.027)$.

CONCLUSIONS: Men between 50 and 70 years have the highest risk of fatal suicide. They prefer to do it at home and with more complicated mechanisms and they always leave a suicide note. Women try to commit more suicides, not under 30 years old and whey they want to be successful they do it on a public place. We found that the older they get the possibilities are higher.

Key words: Consummated suicide, rate, risk factors.

Fecha de recepción: 2.MAY.05

Fecha de aceptación: 8.FEB.06

Correspondencia: Dr. Gabriel Ayala Espinosa. Teléfono de contacto: 609674345. E-mail: gabrielayala@saludalia.com

1 División Medicina Legal y Forense. Facultad de Medicina. Departamento de Patología y Cirugía. Universidad Miguel Hernández. Campus de San Juan. (Alicante). 


\section{INTRODUCCIÓN:}

El suicidio es una de las acciones voluntarias más difíciles de explicar ya que es una conducta que va en contra de la propia existencia. La tasa global de suicidios ha sufrido un aumento importante en las últimas décadas, no sólo en los países desarrollados sino a nivel mundial.

Es un hecho que sigue aumentando con el tiempo aunque más en hombres que en mujeres. Según datos de la Organización Mundial de la Salud (OMS) ya había diferencia en las tasas de suicidio por 100.000 habitantes en 1950, hombres el 16\%, mujeres el 5\%. En 1995 la tasa en mujeres era del $7 \%$, sin embargo en hombres era del $24 \%$.

En el año 2000 (OMS) aproximadamente 1.000 .000 de personas se quitaron la vida, un caso cada 40 segundos, con una tasa de mortalidad global de 16/100.000 habitantes. En los últimos 45 años las tasas de suicidio se han incrementado en un $60 \%$ en el mundo, siendo la muerte por suicidio la $3^{\text {a }}$ causa entre los 15 y 44 años en ambos géneros. Estos datos no incluyen los intentos de autolisis que son unas 15-20 veces más frecuentes que los consumados (en el 2000 entre 10-20.000.000 de personas tuvieron al menos un intento).

En España y según datos de 1998 (OMS, 2000) la evolución de las tasas de suicidio según género y edad es ascendente, a más edad más suicidios; a partir de los 60 años de edad es importante el aumento en ambos géneros, mucho más en hombres que en mujeres. En el grupo de edad mayor de 75 años llega a 25\% y a 10\% respectivamente, encontrando un pico en el grupo de edad de 25 a 35 años.

Comparando con la evolución de las tasas de suicidio a nivel mundial (OMS,2002), podemos pensar a dónde podemos llegar en nuestro país, tasas más altas en grupos de edad mayores de 75 años, tanto en hombres (55\%) como en mujeres (19\%), mucho más acusado en hombres y destacando el aumento de las tasas desde los grupos de edad más joven.

En Dinamarca y Japón el suicidio es la principal causa de muerte en adolescentes. En Europa ha habido un claro aumento en los adolescentes y adultos jóvenes en los últimos 20 años [I].

El fenómeno del suicidio se ha estudiado en varios Partidos Judiciales de la provincia de Alicante como son el de Elda-Villena (1987)[2], Alicante (1987)[3], Orihuela (1992)[4], Elche (2000)[5] y Benidorm (200 I)[6], siendo los suicidios en los hombres los que predominan en todos los municipios.

Pueden influir muchos factores respecto al mecanismo empleado, su elección depende de las influencias socio-culturales, la disponibilidad y accesibilidad de los mecanismos y de los utilizados en intentos previos [7]. Según estudios consultados $[8,9,10,1$ I ] en general el hombre elige ahorcadura, precipitación y arma de fuego, mientras que la mujer suele ingerir una sobredosis de psicotropos o veneno [12].

Simonin (1982), coincidiendo con Durkheim ( 1897), considera que el estar soltero, independientemente del género, duplica la frecuencia de suicidios. Por el contrario, Rodes (1988) encuentra un mayor número de suicidios en casados $(59,8 \%)$ en relación con los solteros $(17,7 \%)[12]$. Para Ruiz Ramos [8] los que más se suicidan son los viudos-as seguidos de los solteros-as y divorciados-as, y en última posición los casados-as.

La diferente ocurrencia del suicidio consumado dependiendo del entorno rural/urbano así como la interacción de esta variable con otras tales como el género y edad ha dado lugar en todo el mundo a gran número de investigaciones [13]. El lugar del suicidio está categorizado en forma 
dicotómica: urbano, si la población de residencia iguala o supera los 100.000 habitantes, o rural si no alcanza dicho número. Las tasas de suicidio urbanas superan a las rurales. La tendencia indica un mayor crecimiento relativo de suicidio en el campo, aunque todavía no se acerque siquiera a las tasas de suicidio de las ciudades. Se describe un aumento del suicidio femenino en edades jóvenes, centrado sobre todo en el entorno urbano y un descenso de la tasa de varones jóvenes de entorno rural $[11,13]$.

En los estudios realizados sobre el suicidio en la provincia de Alicante encontramos un porcentaje elevado de personas que tienen antecedentes de haber atentado contra su vida previamente, desde un 7,47\% en el Partido Judicial de Elda-Villena (1987) hasta el 28,95\% de Orihuela (1992).

Las personas que deciden acabar con su vida pueden dejar algún tipo de documento, generalmente realizado en momentos inmediatamente anteriores a llevar a cabo su acción, haciendo referencia a las causas o motivos que les han empujado. Son de gran valor pero no suponen el cese de las investigaciones ya que son conocidos los casos de homicidios en que se han colocado junto al cadáver o en el lugar de los hechos para simular un suicidio. La presencia de documentos ha originado múltiples estudios de su contenido pero escasos han sido los resultados sobre su frecuencia de aparición y significación clínica.

\section{OBJETIVOS:}

I. Determinar el número de casos de suicidio consumado en San Vicente del Raspeig entre 1990 y 2001.

2. Describir las características, circunstancias y factores de riesgo asociados a los mismos.

\section{MATERIAL Y MÉTODOS:}

Se trata de una investigación con diseño de serie temporal compuesta de un conjunto de diez estudios transversales anuales y consecutivos en el periodo 1990-200।.

Respecto a la metodología de recogida de datos para la realización de este trabajo de investigación se ha utilizado como fuentes de obtención de datos todos los casos de suicidio consumado que se han localizado en los archivos de los Juzgados de Primera Instancia e Instrucción (4 Juzgados) del Partido Judicial de San Vicente, tras la oportuna autorización oficial de la Sra. Juez Decano.

Consideramos que es un periodo lo suficientemente amplio para valorar la incidencia y repercusión de este tipo de muerte en la zona geográfica estudiada y nos permite por otra parte seguir la tendencia que el suicidio ha tenido en los últimos años.

El Partido Judicial de San Vicente situado en la provincia de Alicante lo constituyen 8 municipios que son: Aguas de Busot, Busot, Campello, Jijona, Muchamiel, San Juan de Alicante, San Vicente del Raspeig (cabeza del Partido Judicial) y Torremanzanas. La población media del Partido Judicial es de 88.025 habitantes. Su demarcación es de un 6,98\% de la extensión de la provincia y es un $5,71 \%$ de todos los municipios de la provincia de Alicante.

Inicialmente se indagó sobre la relevancia de ventitrés variables obtenidas a través del estudio de las diligencias previas de los casos de suicidio consumado, seleccionando en el presente estudio 9 variables: género, edad, estado civil, mecanismo, lugar del suicidio, municipio, año del suicidio, intentos anteriores y documentos dejados. 
Debido al método de muestreo en la recogida de datos y al tratarse de un estudio transversal, nos hemos planteado la hipótesis de la independencia de las variables dos a dos. Con objeto de determinar significaciones en dichos contrastes, para tratar de establecer si existe dependencia estadísticamente significativa entre algunas variables seleccionadas, se ha utilizado el test chicuadrado considerando significativos aquellos contrastes con un p-valor menor de 0,05. Se trata de verificar, a partir de una relación entre las variables observadas en la muestra, si dichas variables están o no relacionadas en la población.

Todos los datos de las variables obtenidas se incluyeron en una base de datos del programa informático SPSS (Stadistical Package for Social Sciencies) para Windows versión 10.0.

\section{RESULTADOS:}

Se ha estudiado el suicidio en el Partido Judicial de San Vicente del Raspeig entre los años 1990-200 I encontrándose un total de 78I diligencias previas por muerte, de las que 325 son por mecanismo violento, correspondiendo 185 a accidentes y 92 a suicidios. La tasa general de suicidios en este Partido Judicial es de 10,45 por 100.000 habitantes y año.

\section{Género:}

63 son hombres (68,48\%) y 29 mujeres (31,52\%). La proporción hombre/mujer es de $2,17: 1$.

\section{Edad:}

Entre los 10 y 29 años no se ha producido ningún caso de suicidio en las mujeres. Entre 50-59 años se da el mayor número de suicidios, el $72 \%$ son hombres y el $28 \%$ son mujeres, seguido de la década 70-79 con el 80\% de hombres y 20\% mujeres. Tan sólo entre 60-69 años el número de mujeres supera al de hombres.

En todas las edades encontramos hombres que se suicidan. Los hombres de 50-59 y 70-79 años son los que más consuman los hechos. Mientras que las mujeres son las de la década de los 60 (Figura I).

La relación de las variables género y edad es significativa $(p=0,040)$, los hombres superan en casi todos los grupos de edad. Es factor de riesgo el género masculino y la edad entre 50 y 70 años.

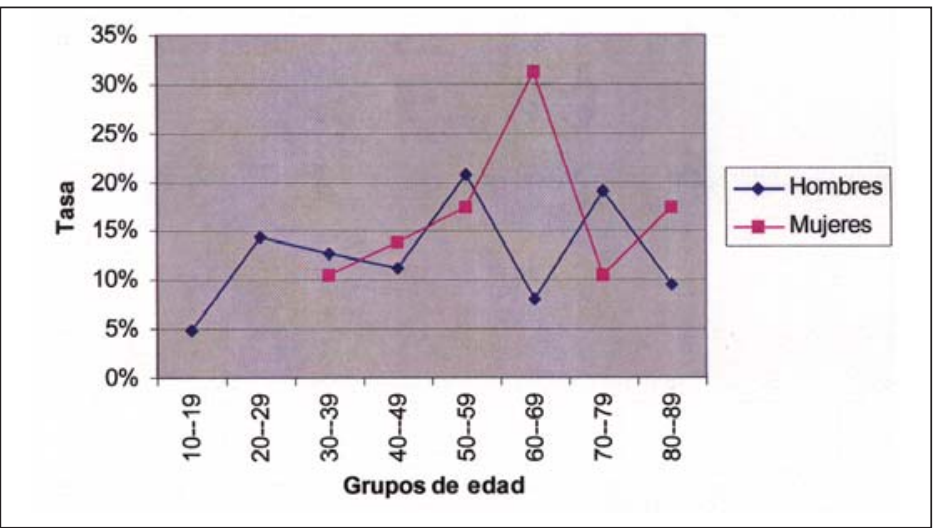

Figura 1. Asociación de las variables género y edad en los suicidios consumados. 


\section{Estado civil:}

En hombres y mujeres que se suicidan el estado civil que encontramos más frecuentemente es el de casado seguido de soltero. No hay diferencias estadísticamente significativas (Fgura 2).

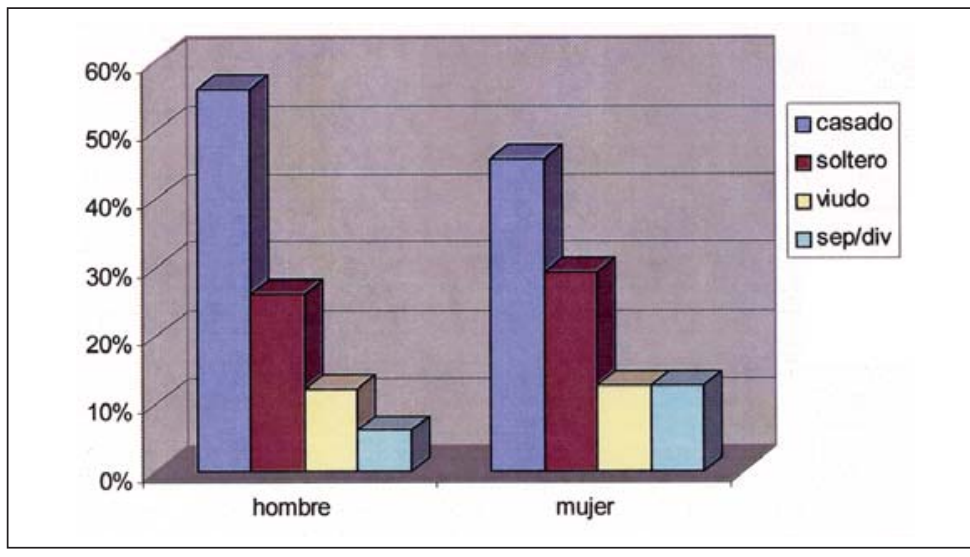

Figura 2. Asociación de las variables género y estado civil en los suicidios consumados.

\section{Mecanismo:}

28 hombres emplean para quitarse la vida la ahorcadura, seguido de la precipitación con II casos; 15 mujeres lo consiguen por precipitación seguido de 5 por ahorcadura.

La ahorcadura, precipitación y atropello por tren son los principales mecanismos empleados por los hombres. Por el contrario, precipitación, ahorcadura e intoxicación cáustica por las mujeres (Figura 3). La relación de las variables género y mecanismo es significativa $(p=0,043)$.

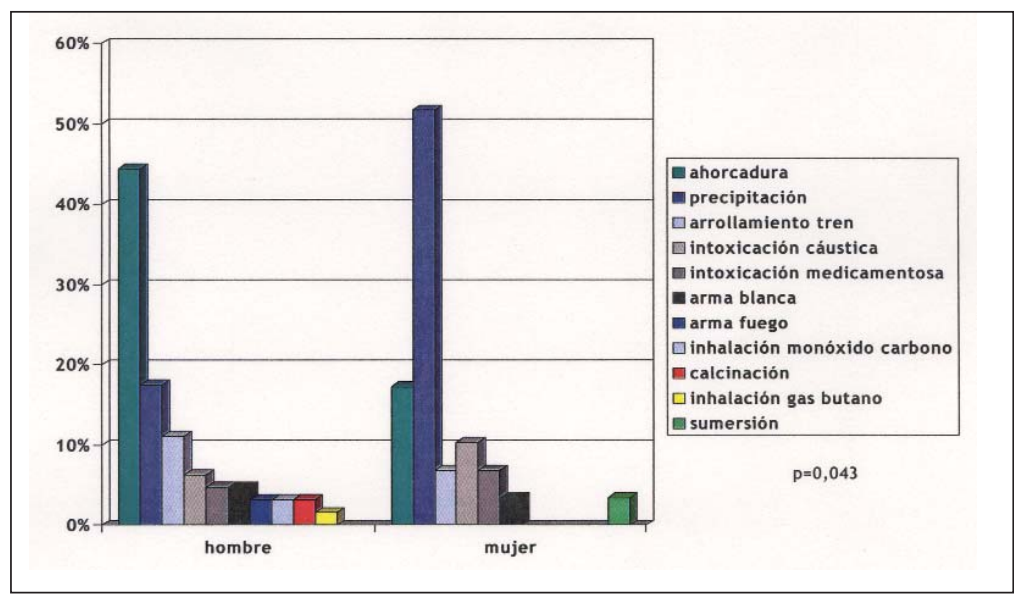

Figura 3. Asociación de las variables género y mecanismo en los suicidios consumados.

\section{Lugar de los hechos:}

El 4I,30\% de los suicidios (38 casos) tienen lugar en su propio domicilio; el 15,22\% ( 4 casos) en la vía pública; y |4, I3\% (I3 casos) en centros hospitalarios.

Los hombres prefieren el propio domicilio seguido del descampado, las vías del tren y centros hospitalarios. Las mujeres la vía pública, domicilio propio y centros hospitalarios. 
De los casos de suicidio acaecidos en centros hospitalarios, los pacientes se quitaron la vida en el Hospital Psiquiátrico de Santa Faz, Hospital Universitario de San Juan, Sanatorio Cardiovascular de San Vicente, Asilos de Ancianos de Jijona, Muchamiel y Campello.

En el propio domicilio se suicidan el $76 \%$ de los hombres y el $24 \%$ de las mujeres, sin embargo, la vía pública es utilizada por el $71 \%$ de las mujeres y el $29 \%$ de los hombres. Tan sólo consuman los hechos hombres en: descampado, lugar de trabajo, supermercado y "otro lugar".

En vías del tren y domicilio familiar predominan los hombres. En centros hospitalarios son 7 mujeres y 6 hombres los que se quitan la vida (Figura 4).

La relación de las variables género y lugar de los hechos es significativa $(p=0,006)$.

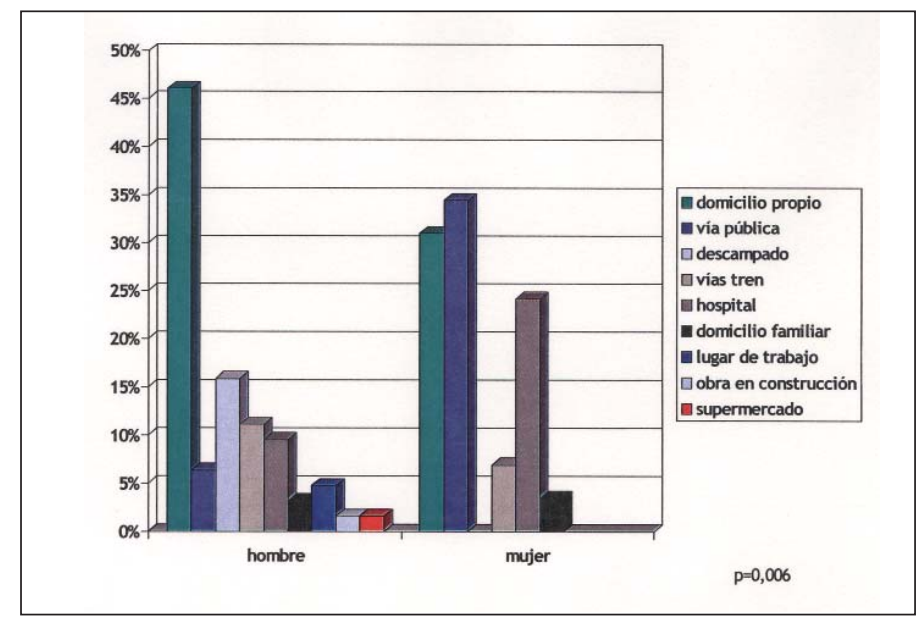

Figura 4. Asociación de las variables género y lugar de los hechos en los suicidios consumados.

\section{Municipio:}

En todos los municipios se quitan la vida mas hombres que mujeres, excepto en Muchamiel en que predominan las mujeres. En Aguas de Busot y Torremanzanas no encontramos ningún caso de suicidio (figura 5). No hay diferencias significativas $(p=0,654)$.

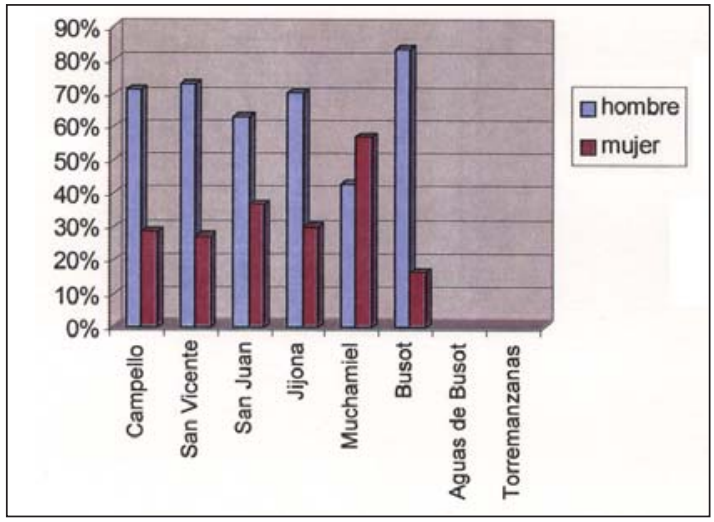

Figura 4. Asociación de las variables municipio y género de los hechos en los suicidios consumados. 
Año:

El mayor número de suicidios en hombres ocurre en 1991, 1999 y 1994 por este orden, mientras que el que menos es 1990. Entre las mujeres el menor número ocurre en 1995, 2000 y 200 I con un solo caso, mientras que el mayor número ocurre en 1991 y 1996 con cuatro. Excepto los años 1990, 1993 y 1996 en que se quitan la vida más mujeres que hombres, el resto de los años estudiados proporcionalmente consuman los hechos más hombres (Figura 6).

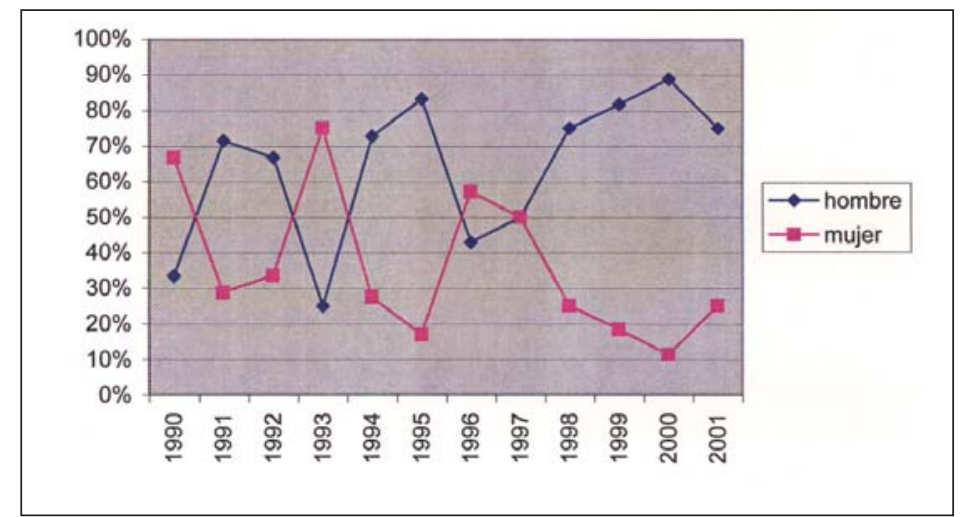

Figura 6. Asociación de las variables año y género en los suicidios consumados.

\section{Intentos previos:}

Los 24 intentos previos de quitarse la vida se han repartido por igual entre los hombres y las mujeres; el hombre ha intentado suicidarse previamente con la misma frecuencia (50\%) que la mujer en los casos que nos consta. Destaca el hecho de que el $92 \%$ de los suicidios en los que no había intentos previos correspondían a hombres (Figura 7). La relación de las variables género e intentos previos es significativa $(p=0,0$ । 0$)$; el número de intentos previos es igual en ambos géneros, si bien el porcentaje es mucho mayor en las mujeres.

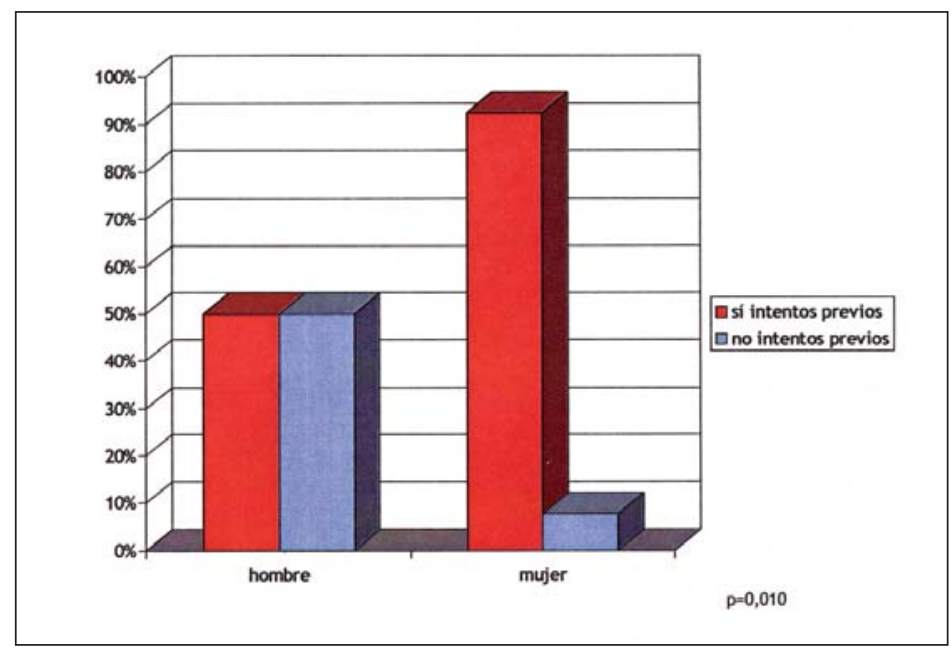

Figura 7. Asociación de las variables género e intentos previos en los suicidios consumados. 


\section{Documentos dejados:}

Los hombres dejan proporcionalmente más documentos que las mujeres.

En los 19 casos de suicidio que dejaron documentos, la mayoría (el 89\%) fueron los hombres y sólo el $11 \%$ las mujeres. La relación de las variables género y documentos dejados es significativa $(p=0,027)$.

\section{DISCUSIÓN:}

Hemos encontrado diferencias estadísticamente significativas en las variables citadas, el género y la edad, género y mecanismo, género y lugar de los hechos, género e intentos previos y género y documentos, lo que significa que el resultado obtenido es real, están relacionadas o muy relacionadas, que hay cierto grado de asociación y que se ha eliminado el posible efecto del azar aunque tenemos el $5 \%$ de probabilidad de error.

Si esta relación es significativa entonces se valora como factor de riesgo. El problema de la validez respecto a toda la población recibe el nombre de significación estadística. Lo que intentamos es acercarnos a la realidad del suicidio. En el resto de variables no hay diferencias estadísticamente significativas lo que significa que el resultado obtenido está ahí, no hay evidencias en su contra, no se puede negar y no existe en la población total la relación observada en la muestra.

En nuestra área de salud es muy importante tener en cuenta los posibles factores de riesgo a efectos de prevención: el que sea una persona mayor sobre todo hombres, el aumento de mujeres en la década de los 60 años, el estar casado, el que los hombres emplean más frecuentemente la ahorcadura y las mujeres la precipitación, el que los hombres prefieren consumar los hechos en su domicilio y las mujeres en la vía pública, la importancia en la prevención de los suicidios que ocurren en los centros hospitalarios, el que tengan antecedentes de intentos previos.

Comparando nuestros resultados con los obtenidos del estudio de otros Partidos Judiciales de la provincia de Alicante: Elda-Villena [2], Alicante [3], Orihuela [4], Elche [5] y Benidorm [6], podemos observar que los hombres son los que predominan en todos los municipios estudiados. En la Figura 8 observamos el género más frecuente del suicida en diversos estudios de la provincia de Alicante.

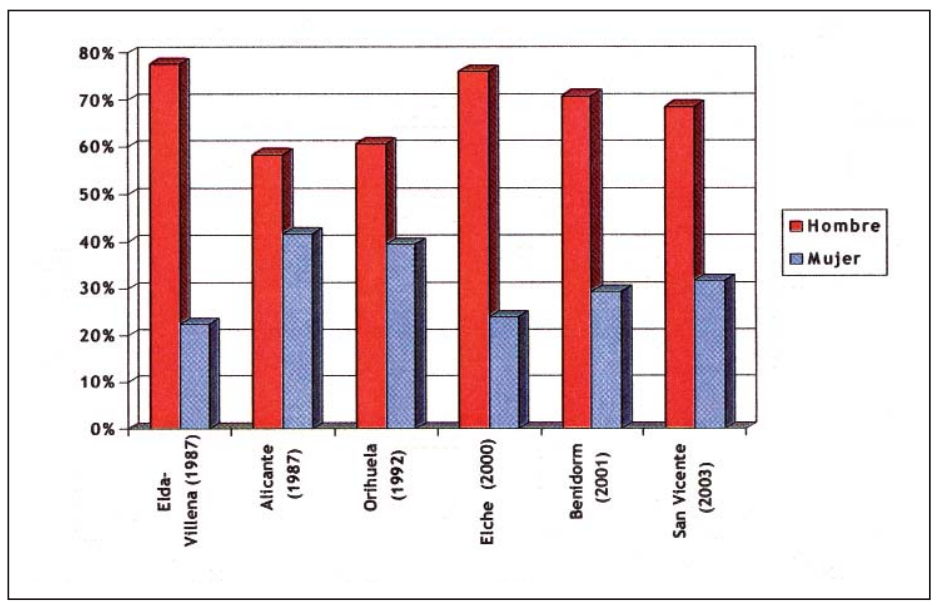

Figura 8. Género más frecuente del suicida en diversos Partidos Judiciales de la provincia de Alicante. 
También es el hombre el que más frecuentemente se suicida según datos de los Institutos Anátomicos Forenses de Granada, Valencia, Palma de Mallorca, Badajoz, Barcelona y en los trabajos sobre este tema realizados en Alcalá de Henares así como para otros autores en Guadalajara [9], Asturias [I 0], Castilla-La Mancha [I I] y en la Comunidad Valenciana [I 4].

Para autores como Balanza [9] y Diekstra [15] el suicidio está entre las diez primeras causas de muerte en grupos de edad de 15 a 34 años, adolescentes y adultos jóvenes del género masculino, tanto en Europa como en EE.UU.

Según Ros Montalban [13] y Vega-Piñero [16] es de destacar el especial dramatismo en los jóvenes de 15 a 24 años, grupo de población en que el suicidio es la $2^{\mathrm{a}}$ causa de muerte después de los accidentes, independientemente del género, y es la $2^{\mathrm{a}}$ causa más común de muerte en Europa en hombres de 15 a 44 años siendo para las mujeres de la misma edad la $4^{a}$ causa de muerte.

En edades más avanzadas el suicidio es sobrepasado por otras enfermedades y deja de estar entre las 10 primeras causas de muerte pero sus tasas aumentan [I I]. Se encuentra mayor incidencia de suicidio consumado entre las personas ancianas, sobre todo hombres [9], siendo las tentativas, ideación y comportamiento, más frecuentes en mujeres, con una razón hombre/mujer de aproximadamente 3 o más en la mayor parte de los países dónde se ha estudiado el fenómeno del suicidio y según autores consultados $[7,8,19,20,21]$, aunque hay países en dónde mueren más mujeres que hombres por esta etiología como China y Kuwait [15].

Para Ruiz Ramos [8] el suicidio actualmente está afectando a población más joven. En los jóvenes se encuentra entre las primeras causas de mortalidad mientras que en los ancianos la importancia radica en su magnitud que llega a ser hasta tres veces superior a la de los jóvenes; tradicionalmente las tasas mayores de suicidio correspondían a las personas de más edad [22].

Comparando con los trabajos consultados no esperábamos encontrar que el estado civil más frecuente es el de casado, aunque si que la ahorcadura como mecanismo de suicidio es más típica de hombres y la precipitación de mujeres. Significativo el encontrar que los hombres prefieran para terminar con su vida su propio domicilio y las mujeres la vía pública, así lo refieren autores como Gómez Alcalde. Otros autores analizan si el suicidio se ha consumado en un medio urbano, es decir dentro de los límites de la localidad, o si por el contrario se ha llevado a cabo en las afueras de la población, buscando descampados o zonas de monte (rural). En este sentido, nosotros encontramos 10 casos en descampado, ( $10,87 \%)$. Coincide con la literatura consultada que en los hombres encontramos menos antecedentes de intentos previos, siendo los que más documentos dejan justificando su acción.

De todo ello se deriva la importancia de conocer su incidencia, las circunstancias que rodean a estas muertes y factores de riesgo necesarios para evaluar el verdadero alcance de este problema y de este modo poder acercarnos a la realidad suicida y poder efectuar una adecuada planificación sanitaria.

\section{LIMITACIONES:}

I. Al tratarse de un estudio de tipo observacional, no experimental, descriptivo y transversal, la ausencia en las diligencias previas de algunos datos de los casos de suicidio puede representar un sesgo importante a la hora de analizar los resultados obtenidos y por ello nos obliga a aplicar el porcentaje válido de cada valor en el análisis de las variables estado civil e intentos previos, eliminando en la comparación la ausencia de información. 
2. Podemos encontrar dificultad en los estudios comparativos por las deficiencias existentes en la epidemiología de la conducta suicida según los datos obtenidos sean del Instituto Nacional de Estadística (I.N.E) o de Institutos Anatómico-Forenses y/o Juzgados.

\section{CONCLUSIONES:}

I. La tasa general de suicidio en el Partido Judicial de San Vicente es superior a la encontrada en otros estudios consultados de zonas geográficas de la Comunidad Valenciana. Según nuestros resultados, y la bibliografía consultada, el género masculino se asocia a un riesgo más elevado de suicidio.

2. La mayor incidencia de suicidios acontece a partir de los 50 años (63\%), no encontrando ningún caso en menores de 18 años. Los hombres que más frecuentemente se quitan la vida son los de la década de 50 y de 70, excepto en década de 60, 80 y 40 en que es más frecuente la mujer. No encontramos ninguna mujer menor de 30 años que se suicidara. Los hombres utilizan preferentemente la ahorcadura y en segundo lugar la precipitación; las mujeres prefieren la precipitación y como segundo mecanismo la ahorcadura. Las mujeres eligen la vía pública, el domicilio propio y centros hospitalarios (las mujeres se suicidan más que los hombres en centros sanitarios). Los hombres el domicilio propio, el descampado (son los únicos que lo eligen), vías del tren (más frecuente en hombres), lugar de trabajo, obra en construcción y supermercado. En casi todas las mujeres que se suicidaron constaban antecedentes de intentos previos, mientras que en los hombres sólo en la mitad. Por el contrario, los hombres son los que con más frecuencia dejan constancia por escrito de sus motivos para quitarse la vida.

3. Teniendo en cuenta las limitaciones de las estadísticas oficiales a la hora de valorar el fenómeno suicida, por la infranotificación que se produce y las dificultades metodológicas que presenta la comparación de las tasas registradas, consideramos los datos forenses y el estudio de las diligencias previas judiciales una fuente precisa para el análisis de las estadísticas sobre el suicidio consumado.

\section{BIBLIOGRAFÍA:}

I. Tondo Leonardo, Baldessarini Ross. Suicide: desciptive and epidemiological considerations. www.medscape/com. 17//2/2002.

2. Rodes Lloret F. Estudio del suicidio consumado en los Partidos Judiciales de Elda y Villena. Años 1977-1986. Tesis Doctoral. Universidad de Alicante, 1987.

3. Pamblanco Lillo E, Rodes Lloret F, Martí Lloret JB, Navarro López MI. Incidencia del suicidio consumado en mayores de 60 años. Rev Iberameric de Geriatría y Gerontol 1990; 6 (5):69-72.

4. Giner S. Estudio del suicidio consumado en el partido judicial de Orihuela, 1982-1991. Tesis Doctoral. Universidad de Alicante, 1992. 5. Alberdi IM. El suicidio en el Partido Judicial de Elche (19901998) y estudio de las notas suicidas. Trabajo de investigación para la obtención del Título de Magíster Universitario en Criminología del Instituto de Criminología de la Universidad de Alicante, 2000.
6. Chiarri Rodrigo JM. Estudio médico-legal del suicidio consumado en el Partido Judicial de Benidorm durante la década 1990-1999. Tesis Doctoral. Facultad de Medicina. Universidad Miguel Hernández. Alicante, 2001.

7. Ruiz Doblado S. Intentos de suicidio en un área rural. Conducta suicida y factores socioculturales. An Psiquiatría 2000; 16:57-62.

8. Ruiz Ramos M, Muñoz Bellerin J, Ramos León JM, Gil Arrones J, Ruiz Pérez J, Muriel Fernández R. Tendencia de la mortalidad por suicidios en Andalucía desde 1976 a 1995. Gac Sanit 1999; 13:135-140.

9. Balanza Soriano J, Chamorro García L. Análisis del suicidio consumado en Guadalajara. Aspectos epidemiológicos y perfiles suicidas. Arch Neurobiol 1999; 62 (2): I63-176. 
10. Iglesias C, Alvarez JA. Un estudio del suicidio en Asturias: Incremento de la frecuencia en las dos últimas décadas. Actas Esp Psiquiatr 1999;27(4):217-222.

II. Morant C, Criado-Alvarez JJ, García Piña R, García Guerrero J, Domper Tornil J. Mortalidad por suicidio en Castilla-La Mancha (1991-1998). Psiquiatría biológica 200I; 8 (4):I35-I40.

12. Dorado E. La mortalidad violenta en el Partido Judicial de Cambados (1989-1998). Tesis Doctoral. Madrid. Universidad Complutense. Facultad de Medicina; 2001.

13. Ros Montalbán S, Hernández Vidal P, Bulbena Vilarrasa A. Depresión y ansiedad asociada. Ed. Mayo. Barcelona; 2002,pp 69-84. 14. Pérez-Hoyos S, Fayos G. Evolución de la mortalidad por suicidio en el País Valenciano 1976-1990. Gac Sanit 1996;10:4-II.

15. Diekstra RFW, Garnefski N. On the nature, magnitude and causality of suicidal behaviours: an international perspective. Suicide and Life-Threatening Behaviour 1995;25(I):36-57.

16. Vega-Piñero M, Blasco-Fontecilla H, Baca-García E, Díaz-Sastre C. El suicidio. Salud Global 2002;4:I-15.
17. De Leo D, Coforti D, Carollo G. A century of suicide in Italy. A comparision between the old and the young. Suicide life threat behaviour 1997;27:49-239.

18. Pedros Rosello A, Vera Albero P. Análisis epidemiológico de la conducta suicida en el área 13 de Valencia. An Psiquiatría 2001;17:48-52.

19. Mirón JA, Saenz MC, Blanco L, Fernández C. Epidemiología descriptiva del suicidio en España (1906-1990). Actas Luso Esp Neurol Psiquiatr Cienc Afin 1997; 25(5):327-331.

20. Peña I, Martín C, Gutiérrez JL, Carpintero MJ, Torija C, Alvarez JR. Estudio epidemiológico del suicidio consumado en la Comunidad de Madrid. An Psiquiatría 1997;13(8):320-331.

21. Gómez Alcalde MS, Reyes Martín A. Suicidio consumado y enfermedad mental previa; aproximación epidemiológica. Psiquis 1996;17(8):402-409.

22. Gómez Alcalde MS. Mortalidad violenta en el Partido Judicial de Alcalá de Henares. 1985-1989. Tesis Doctoral. Facultad de Medicina. Universidad de Alcalá de Henares, 1993. 